\title{
Helpers Have Little to Laugh About: Group Structure and Vocalisation in the Laughing Kookaburra Dacelo novaeguineae.
}

\author{
Heinz-Ulrich Reyer \& Dieter Schmidl \\ Max-Planck-Institut für Verhaltensphysiologie, Abt. Wickler D-8131 Seewiesen, FRG
}

Received 27 July 1987, accepted 26 November 1987

\begin{abstract}
Summary
Reyer, Heinz-Ulrich \& Schmidl, Dieter (1988). Helpers have little to laugh about: Group structure and vocalisation in the Laughing Kookaburra Dacelo novaeguineae. Emu 88, 150-160.

Laughing Kookaburras Dacelo novaeguineae combine a conspicuous group vocalisation with a social structure in which subordinate helpers assist a dominant breeding pair in raising young. The structural and temporal characteristics of the laugh song in captivity and in the field were related to spatial distribution of group members, to social interactions within and between groups and to group size and territory size. Under all conditions, group songs lasted longer than pair songs and contained a higher proportion of the loudest element, the actual laugh part. Other parameters of song structure did not differ either with group composition or context. Joint songs fell into two categories: those during the day, (usually involving the breeding pair only); and those at the roost during dusk and dawn, usually involving all group members, i.e. the pair plus one to three helpers. Songs without the breeding pair or with only one member of it were rare. The spatial distribution of group members during singing was almost identical to that when not singing, but differed from a random distribution. Songs at the roost amounted to about $60 \%$ of all songs produced and shifted with the times of sunrise and sunset. At dusk, song frequency was mainly determined by group size and at dawn mainly by the total number of neighbours. During daytime, singing was usually connected with inter- and intra-group disputes. We conclude that the Kookaburras's laugh song signals aggression and aids in (a) defending a territory, (b) guarding the mate and (c) establishing and maintaining a dominance hierarchy between breeders and helpers. In this last context (c) helper singing, especially during daytime, indicates a challenge to a breeder's status.
\end{abstract}

\section{Introduction}

The song of the Laughing Kookaburra Dacelo novaeguineae Hermann is well known and has become the subject of many Australian Aboriginal beliefs, of stories told by the early white travellers, of folksongs and even radio shows (Parry 1972).

The first scientific study of its vocal repertoire was by Parry $(1968,1972)$. The same author showed that Kookaburras are co-operative breeders with progeny from previous broods helping their parents to raise subsequent young (Parry 1968, 1973). These helpers (or 'auxiliaries' as she named them) are subordinate to the breeding pair in an age- and sex-related hierarchy. Hierarchies have also been reported for other co-operative breeders. In a few species they lead to rank-related reproduction and in most others, including Kookaburras, to the exclusive reproduction of a dominant pair (see Reyer et al 1968 for a discussion of the relationship between dominance and 'reproductive suppression' and for further references). Often, the skew in reproduction (Vehrencamp 1983) itself is used as a measure of dominance, with no independent measure of behaviour provided. In such instances, any attempt to relate hierarchies to fitness becomes a tautology. In many cases it may not be easy to find an independent measure of dominance. Where hierarchies are stable, overt aggression is usually rare and more subtle signs of conflict have to be used. This, however, first requires analysis of the meaning of various behaviour patterns.

In this paper we analyse the meanings of the laugh song and relate them to the dominance structure in Kookaburra groups. Information was derived from: (1) group size and territory size, (2) structure of laugh songs, (3) annual and (4) diurnal pattern of songs, (5) group composition during singing, (6) spatial distribution of group members when not singing and (7) social interactions within and between groups.

\section{Methods}

\section{Analysis of vocalisations}

Songs were recorded with a directional microphone (AKG C8) and a tape recorder (Nagra $\mathrm{SN}$ ). Recordings were then transformed into sonagrams on a Nicolet Ubiquous UA 500 spectrum analyser. Seven elements were identified (Fig. 2); their names and verbal descriptions have been adopted from Parry $(1968,1972)$, unless stated otherwise:

(1) Song: 'a full throated, boisterous laugh' (Parry 1972), which in this paper is also referred to as laugh song or just laughing. The song is composed of the actual laugh part, a series of loud ha ha ha' sounds and some of the following elements (2)-(6). 
(2) Kooaa: a short, guttural sound, which can be given alone, often repeatedly, but also as an introduction to the song.

(3) Rolling: a fast repetition of identical syllables; the rolling can last several seconds and is often, but not always, followed by a laugh song.

(4) Gurgle: resembles the rolling but has longer pauses between the syllables and an emphasis on a lower harmonic frequency. Parry $(1968,1972)$ apparently subsumed the 'rolling' and the 'gurgle' under 'chuckle' but we found them distinguishable even by ear.

(5) Gogo: loud, distinct syllables, sounding like 'gogo...'. It may be equivalent to what Parry (1968, p. 48) describes as "who who who', which in her study was given too infrequently to examine.

(6) Cackle: a harsh, loud, repetitive sound, similar to the "haha' component of the laugh song but with a short 'a' (Parry 1968, p. 46).

(7) Squawk: 'a hoarse, monosyllabic sound, low in pitch' (Parry 1968, 1972). In this study no distinction was made between the 'squawk' and the 'soft squawk', which is very similar but slightly lower and softer in tone (Parry 1968, 1972).

Volumes of the different song elements were measured from tapes in relation to the lowest element, the 'gurgle', using an EZGA2 sound level meter (Rohde \& Schwarz, Munich).

\section{Captivity study}

A pair of Laughing Kookaburras was obtained from the 'Vogelpark Walsrode' (West Germany) in October 1982 and kept in an $8.1 \times 2.7 \times 3.0 \mathrm{~m}$ aviary, which included a heatable indoor area of $3.1 \times 2.7 \times 2.9 \mathrm{~m}$ (length $\times$ width $\times$ height). The birds bred and raised one male offspring in June 1983. In June 1985 two were returned to the Vogelpark Walsrode after the offspring had killed his mother. For details about keeping, feeding and breeding Kookaburras in captivity see Schmidl (1985).

From June to October 1983 and from May to July 1984 the behaviour of each parent was quantified twice a week during two 15 min sessions per day: one in the morning and the other in the afternoon. In each session, one focal bird was observed and its behaviour at the onset of every minute was written down ('instantaneous sampling', Altmann 1974). Among the eight recorded variables $(\mathrm{a}-\mathrm{h})$ listed below three, $(\mathrm{e})-(\mathrm{g})$, are vocalisations as described above. (a) CP: change of perch as a measure for mobility. (b) EN: entering nest-hole. (c) IC: incubating eggs. (d) FY: feeding young before and after fledging. (e) SG: song (f) $\mathrm{KO}$ : 'kooaa' (g) SQ: 'squawk' (h) DS: distance between the focal animal and its mate in categories of $0,25,50,100,200,300, \ldots, 700 \mathrm{~cm}$, whichever was nearest. As male and female were observed at different $15 \mathrm{~min}$ intervals, their DS is not necessarily the same.

At the end of the two daily 15 min sessions, the frequency of each behaviour pattern was counted (possible maximum $=30$ ) and distances were averaged over $30 \mathrm{~min}$. This yielded one daily frequency for each behaviour pattern (possible maximum $=30$ ) and one average distance per bird. Frequencies and average distances were obtained for each parent on 55 observation days. They were standardised by expressing the daily value of each variable as a percentage of the grand total of that variable, summed over the 55 days. The distributions of these standardised values did not differ between the parents for any of the eight variables (Kolmogorov-Smirnov test). Therefore, standardised values were averaged for male and female.

\section{Field study}

A four week field study was conducted from September 10 to October 7, 1983, near Armidale, NSW, Australia $\left(30^{\circ} 31^{\prime} \mathrm{S}, 151^{\circ}\right.$ $40 \mathrm{E}$ ). Sixteen Kookaburra groups of two to five birds were observed, eight of them on a regular time schedule $(0400-1900$ h) for one or two days each.

Birds in groups of two and three could be individually recognised from plumage differences alone. For the groups of four and five, birds were caught in spring traps baited with grasshoppers or earthworms, and then marked with coloured leg bands and by dyeing parts of the plumage. These markings, together with the natural variation in unmarked birds, allowed identification of all group members.

Parry's (1973) key of plumage characteristics was used to determine sex and breeding status of group members. Birds with vivid blue rumps and white feathers above the eyes were termed 'breeding males' $(\mathrm{m})$; and birds with brown rumps and buff above the eyes were termed 'breeding females' (f). Parry (1973) never found an adult female with a blue rump, but $15 \%$ of her adult males had brown rumps $(n=26)$. In our population, however, each group contained at least one blue-rumped bird. Therefore, sex identification among breeders was unambiguous. The name 'breeder' was applied even though at the time of the study none of the pairs had eggs or young, but nesthole inspection, courtship feeding and copulations in some groups signalled the reproductive season ahead. All other birds in the group were termed 'helpers' (h), because Parry (1973) has shown that only one pair per group breeds and that supernumerary members assist that pair in raising young. Helpers resembled the breeders, mainly the females, but usually had duller and slightly darker feathers. Status identification based on plumage was unambiguous in all groups but one. This exceptional group contained two birds with bright blue rumps. However, courtship feeding and copulations could to be used to tell breeder and helper apart (Perry 1968).

Perching positions and flying paths were recorded on two topographic maps (published by the Department of Lands, Sydney) with scales of 1:31880 (2 inches to 1 mile; Armidale 9236IV-N) and 1:25000 (Dumaresq 9237-III-S), respectively. This yielded an average of 41 perching positions per group with a minimum of 29 in a group of two and a maximum of 51 in a group of four. For each Kookaburra group, the connecting lines of the outermost positions were taken as territory boundaries (maximum polygon method). Territory size was defined as the area within these lines.

Other details of data collection are given with the respective results. Statistical analysis was done with SYSTAT, Version 3.0, run on an EPSON-QX 10 personal computer. Non-parametric tests were made when data differed significantly from a normal distribution and parametric tests when they did not (Lilliefors' modification of the Kolmogorov-Smirnov test with $P=0.10$ as a decision rule; Sachs 1984).

\section{Results}

\section{Group size and territory size}

Of the 16 groups in the field, eight $(=50 \%)$ were pairs without helpers. The other half was made up of four groups 
with three birds each, one group with four birds and three groups with five birds each. This gave a total of 47 birds, 15 of which were helpers $(=32 \%)$. Similar figures are reported by Parry $(1973$, p. 84 ) for her population at Belgrave, Victoria, where helpers amounted to $34 \%$ of all birds $(n=67)$ and groups with helpers to $54 \%$ of all groups $(n=22)$.

Territory size, measured for only nine of our 16 groups, correlated positively with group size (Fig. $1 ; r_{\mathrm{s}}=0.73, n=$ $9, P<0.05$; Spearman rank correlation, 2 -tailed). The average area occupied per bird was 8.75 ha (median; interquartile range 5.29-9.84). It appears from Figure 1 that the increase in territory size becomes smaller with each additional bird and finally would reach zero, thus leading to a maximum territory size. However, the data were insufficient to test whether a curve or a linear fit was better.

\section{Structure of laugh songs}

The song elements A total of 83 songs with known parti-

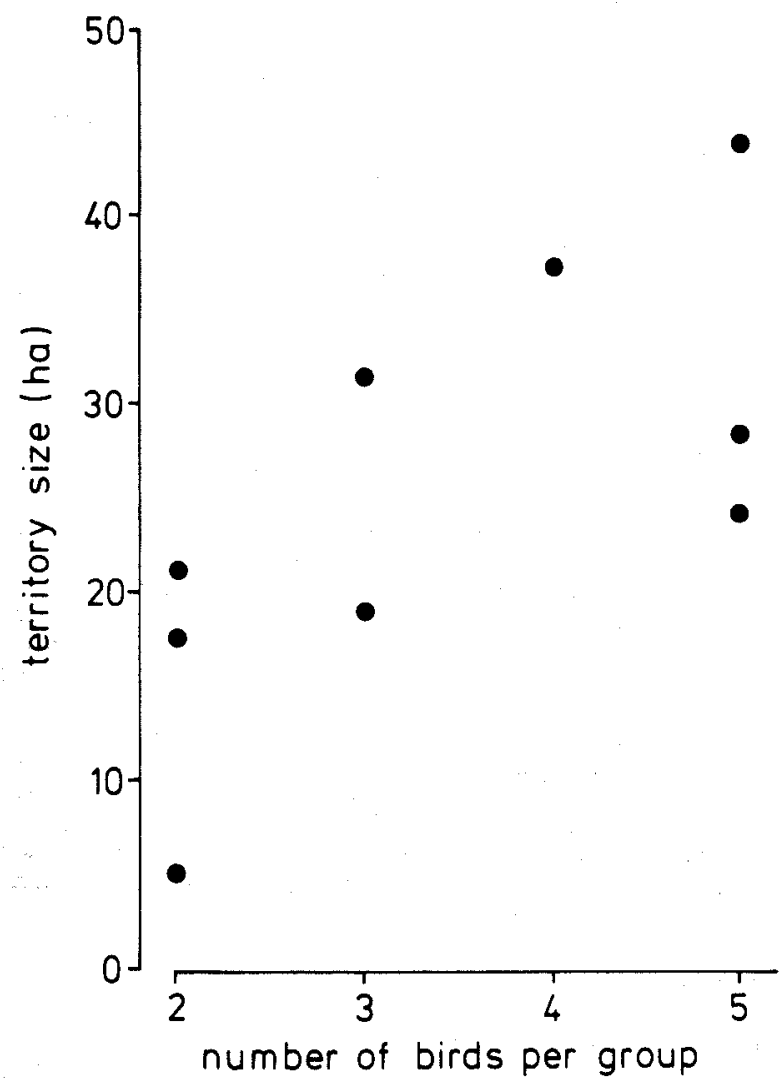

FIGURE 1 Relationship between number of birds per group and size of the group territory in ha. cipants were tape-recorded and sonagraphed: 65 from 11 groups in the field and 18 from the group in captivity. It proved almost impossible to tell from the sonagrams which bird produced which song elements, even when only two participants were involved. With three or more birds, the overlap in frequencies resulted in little more than a confusing blur of black and grey.

To overcome this problem, the captive birds were separated; two birds were confined to the indoor part of the aviary ( $m-f, m-h$ or $f-h)$ and the remaining one was confined to the outdoor part ( $h, f$ or $m$ ). Songs were then elicited by playbacks. The partition allowed the Kookaburras to hear each other and sing together, but guaranteed that vocalisations of the two indoor birds were not superimposed onto the tape-recording of the single outdoor bird. Typical examples of the resulting sonagrams are shown in Figs $2 a$ (male) and $b$ (female).

The songs of both sexes contain five elements, four of which are very similar for males and females. These are the 'kooaa', the 'rolling', the 'laugh' and the 'cackle' (Fig. 2). The fifth element seems to be sex specific. In 18 songs produced by isolated males ( 16 by the mated male, two by the helper), all laugh parts were followed by the 'gogo...' (Fig. 2a), whereas in 14 songs produced by the isolated female all laugh parts were followed by the 'gurgle' (Fig. 2b). Sex-specificity is also indicated by three songs involving the two captive males only: all three contained the laugh-gogo transition, but lacked the gurgle. Similar data from the field are not available, partly because singing alone hardly ever occurred and partly because the sex of most helpers was unknown, making it impossible to compare male-male and female-female songs; there also may be dialects. Therefore, results from the captive group should not be generalised, but they suggest that the 'gogo' is confined to males and the 'gurgle' to females.

The sequence of elements depicted in Fig. 2 is typical but many other sequences also occur. Some elements may be omitted altogether (especially 'kooaa', 'rolling' and 'gogo'). Elements other than the 'kooaa' may initiate the song (particularly the 'cackle' and 'rolling') and partial

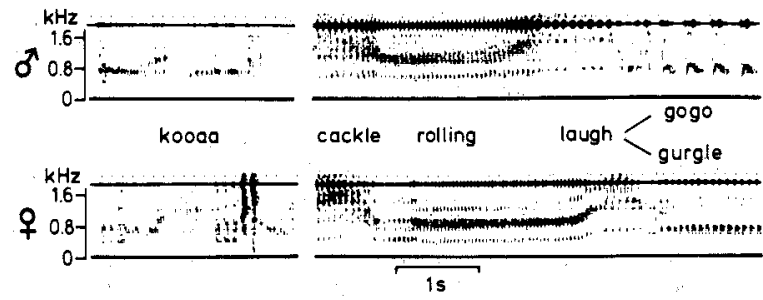

FIGURE 2 Typical sonagrams of male (a) and female (b) song elements, including their names. For further explanations see text. 
sequences like 'rolling-laugh' or 'laugh-cackle' can be repeated several times within one song. Moreover, at each stage of the song different participants usually use different elements (e.g. male: 'cackle', female: 'gurgle') rather than singing in unison, and repetition rates of elements may differ (cf. 'cackle' of males and females in Figs 2a and 2b).

Song structure in relation to participants. The high variability and mixing of elements plus the considerable overlap in sound frequencies makes sonagrams of pairs and groups difficult to read. However, once the elements had been identified in single birds, it was possible to spot
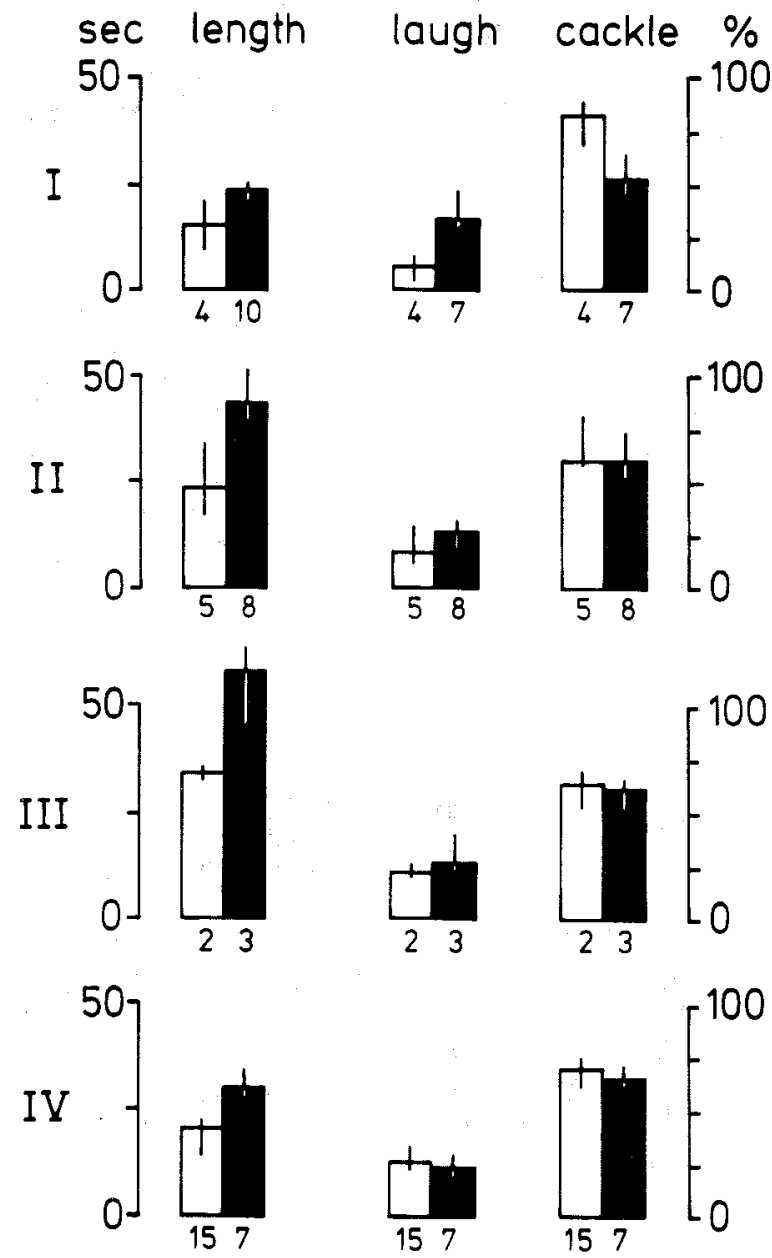

FIGURE 3 Average lengths of songs (sec) and average time proportions of cackle and laugh elements $(\%)$ in four situations: $\mid$ = evening roost, $\|$ = morning roost \|\| = daytime unprovoked, $N=$ daytime, provoked. White bars are for malefemale songs, black bars are for songs involving the mates plus helper(s). Medians with interquartile ranges are given. Numbers below the bars denote sample sizes. them in songs by two or more Kookaburras by carefully listening to the tapes. The following 14 measurements (a$\mathrm{m})$ were taken from 54 recordings: the proportion of time for (a) 'kooaa', (b) 'rolling', (c) 'gurgle', (d) 'gogo', (e) 'cackle' and (f) the actual 'laugh' part in relation to $(\mathrm{g})$ the total duration of the song; the relative volumes of $(\mathrm{h})$ 'kooaa', (i) 'rolling', (j) 'gogo', (k) 'cackle' and (l) the 'laugh' part in relation to (m) the absolute volume of the 'gurgle'.

The 'kooaa' element $(\mathrm{a}, \mathrm{h})$ was excluded from the analysis altogether because (1) it is usually the first element in a song, alerting the observer to switch on the tape but often escaping the recording; (2) it does not necessarily initiate a song - many times no other elements follow it; and (3) pauses between single 'kooaas' are highly variable. Consequently, it is too rare and too unreliable to define the beginning and length of a tape-recorded song.

The absolute volume of the 'gurgle' (m) was also excluded from the analysis because it is influenced by distance, vegetation, recording level and other parameters independent of the song itself. It was only measured to provide a reference for the relative volumes of other vocalisations. Two of these relative volumes, those of 'rolling' (i) and 'gogo' (j), also could not be analysed

TABLE 1 Song parameters in relation to group composition and context

\begin{tabular}{llrrr}
\hline Variables & $R^{2}$ & $d f$. & F-ratio & \multicolumn{1}{c}{$P$} \\
\hline DURATION & 0.501 & & & \\
composition & & 1 & 17.44 & $<0.001$ \\
context & & 3 & 6.19 & 0.001 \\
$\begin{array}{l}\text { composition * } \\
\quad \text { context }\end{array}$ & 3 & 0.53 & 0.663 \\
\hline LAUGH & & & & \\
composition & 0.348 & & & \\
context & & 1 & 9.85 & 0.003 \\
composition * & & 3 & 0.38 & 0.768 \\
$\quad$ context & & 3 & 4.349 & 0.009 \\
\hline CACKLE & & & & \\
composition & 0.195 & & & \\
context & & 1 & 3.00 & 0.090 \\
composition * & & 3 & 0.34 & 0.795 \\
$\quad$ context & & 3 & 2.21 & 0.100 \\
\hline
\end{tabular}

Results of two-way analyses of variance (ANOVA) testing for the effects of group composition, context of singing and of their interaction composition * context (= independent variables) on each of the three song parameters 'duration', 'laugh' and 'cackle' (dependent variables). $R^{2}=$ coefficient of determination; $d f$. = degrees of freedom. Only independent variables with $P<0.05$ can be considered to influence the song parameters. 
because superimposed louder elements prevented volume measurements in sufficient numbers.

Each of the remaining eight variables was subjected to a two-way analysis of variance (ANOVA) with four categories of contexts (evening roost, morning roost, daytime and playback response) and two categories of group composition ( $m-f$ and $m-f-h)$. The other three possible combinations (m-h, $\mathrm{f}-\mathrm{h}, \mathrm{h}-\mathrm{h})$ were not considered as they were rare (Fig. 7).

Total duration of the song (variable g, above) depended on both context and group composition (Table 1 and Fig. 3 ). Songs by pairs lasted longest during daytime, followed in descending order by morning roosts, evening roosts and playback response. Songs by groups followed the same sequence but were longer than pair songs in all contexts ( $P$ $<0.05$ for all pairwise comparisons, Mann-Whitney Utest, 2-tailed).

The proportion of the actual 'laugh' part (f) tended to be higher in group than in pair song, but also depended on the context (Table 1 and Fig. 3). In pair songs, the proportion decreased from playback response through daytime and morning roost to evening roost songs. In group songs the sequence was exactly reversed. Also, for the 'cackle' (e), too, there tended to be differences between pairs and groups, but in different ways in different contexts (Table 1 and Fig. 3); results were not significant and less consistent. Overall, however, the 'cackle' proportion decreased with increasing laugh proportion $(r=-0.555, d f .=49, P<$ $0.001)$, as did the 'gurgle' proportion $(r=-0.299, d f .=49$, $P<0.05$ ). All other pairwise comparisons of element proportions were not significant.

Also, the other variables (relative duration of 'rolling',
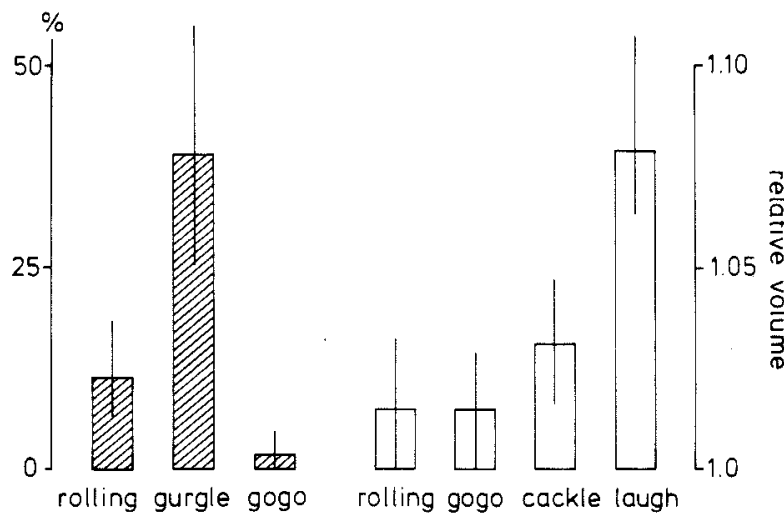

FIGURE 4 Medians and interquartile ranges for song variables not differing with group composition and/or context. The durations of roll, gurgle and gogo are given as percentages of total song duration; the volumes of cackle and laugh are expressed in multiples of the absolute gurgle volume. 'gurgle' and 'gogo' and relative volume of 'cackle' and 'laugh') did not differ significantly either with context or with group composition. Therefore, data for each of these five variables were pooled and medians and interquartile ranges calculated over all categories (Fig. 4). Note that the percentages of the various elements do not necessarily add up to $100 \%$. The sum may be much higher because different birds may sing different elements at the same time.

\section{Songs in relation to breeding cycle}

Data on the song pattern in relation to the breeding cycle could be collected only from the captive group. Figure 5 relates song pattern to the patterns of two other vocalisations ('kooaa' and 'squawk'), three reproductive patterns (entering nesthole, incubating and feeding young), one mobility pattern (change of perch) and one distance measure.
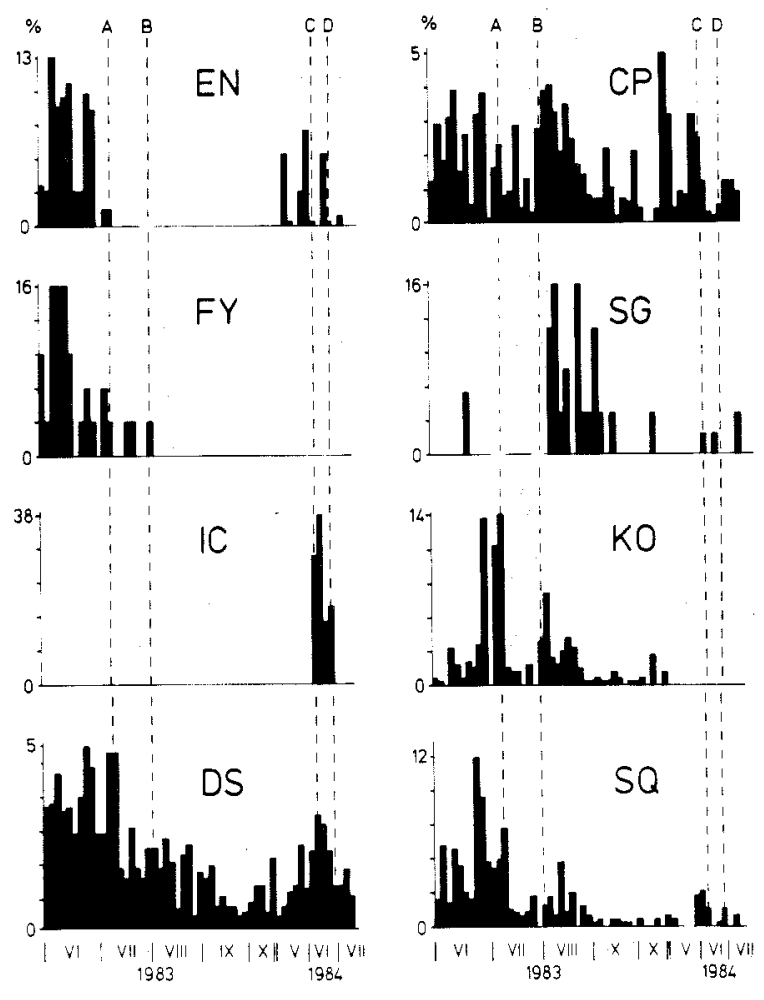

FIGURE 5 Average frequencies and annual distributions of eight behavioural patterns measured in the captive group. Distributions were averaged over both sexes and standardised for all variables. $E N=$ entering nest-hole, $F Y=$ feeding young, $I C=$ incubation, $D S=$ distance between mates, $C P=$ change of perch, $S G=$ laugh song, $K O=$ 'kooaa', $S Q=$ 'squawk'. Broken lines denote dates of fledging $(A)$ and independence $(B)$ of young and of start $(C)$ and termination $(D)$ of incubation. For more detailed descriptions of variables see Methods' and for further explanations see text. 
There were three periods of high mobility (Fig. 5, CP). Two were contemporary with, and caused by, the adults' frequent visits to the nesthole during inspection, incubation and feeding (June 1983 and May 1984; Fig. 5, EN, FY, IC). As the nestbox was some distance from the perches and only one bird at a time could be close to it, these activities also resulted in relatively large distances between the mates (Fig. 5, DS). The third activity peak coincided with the main laugh song peak (Fig. 5, SG), which rose one week after the parents had stopped feeding the fledged young and then lasted for about five weeks (11 August19 September 1983).

This period also overlapped with small peaks in two other vocalisations, the 'kooaa' and the 'squawk' (Fig. 5, $\mathrm{KO}, \mathrm{SQ}$ ). The main peak in 'kooaa' was around fledging. Thus we agree with Parry $(1968,1972)$ that the 'kooaa' is a warning and attention-seeking device. In this case it probably helps the young enter a new, unknown environment. The main peak of the 'squawk' preceded fledging; another small peak coincided with incubation (Fig. 5, IC). These two peaks support Parry's $(1968,1972)$ findings that adults only squawk in the breeding season and stop when the young fledge. She interprets the sound as a food begging and aggression reducing call. Thus a third 'squawk' peak, lying between the other two, may indicate some interest in a second breeding attempt immediately after the first young became independent. In summary, the peak in laugh song activity did not overlap with any of the nest activities such as hole inspection, incubation or feeding young (Fig. 5, EN, IC, FY); it did coincide, however, with a period of high mobility (Fig. 5, CP). attentiveness (Fig. $5, \mathrm{KO}$ ) and probably some tendency to breed (Fig. 5, SQ). In the field this period is characterised by 'trapeze displays' and 'circle flights' (Parry 1972).

\section{Diurnal pattern of songs}

In eight territories the times and frequencies of song were recorded from 0400 to $1900 \mathrm{~h}$ on one or two days each. The resulting daily distribution is plotted in Figure 6. There are two peaks, one between 0430 and $0530 \mathrm{~h}$, the hour preceding sunrise and the other between 1730 and 1830 $\mathrm{h}$, the hour of sunset. Between these peaks, songs were extremely rare in most groups and at most hours. Only in two cases did birds laugh frequently during daytime. One case involved a pair entangled in a boundary fight with neighbours between 1630 and $1730 \mathrm{~h}$. This resulted in $33.3 \%$ of all songs during that hour $(n=30)$. The other case comes from a group of four in which the mated male quarrelled and fought with one of his helpers for several hours.

During the dawn and dusk peaks most songs occurred at the roosts or close by, soon after the birds left them or

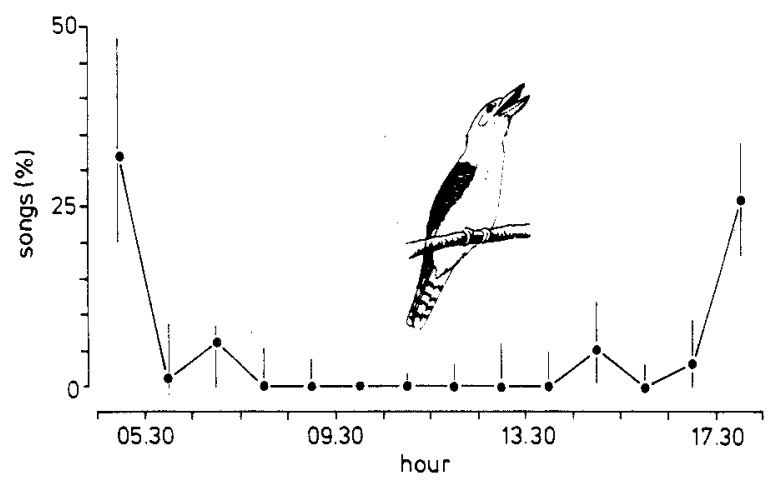

FIGURE 6 Daily distribution of songs. The number of songs in a particular hour is plotted as the percentage of all songs between 0400 and $1900 \mathrm{~h}$. Values are medians of eight territories; inter-quartile ranges are indicated by vertical lines.

before they visited them. This spatial and temporal clumping of songs provided sufficient data to allow a more detailed analysis of the factors determining their timing and frequency. Four multiple regression analyses were performed with the following dependent variables: (a) time of first song in the morning, (b) time of last song in the evening, (c) number of songs at dawn and (d) number of songs at dusk. The independent variables were: (1) group size, (2) territory size, (3) number of neighbouring groups and (4) number of neighbouring birds. The fifth variable, which was included as an independent one, varied with the type of analysis: in analyses (a) and (c) it was the time of sunrise, in analyses (b) and (d) the time of sunset.

With increasing daylength, the time of the first song in the morning advanced and the last laughter in the evening occurred later (Tables $2 \mathrm{a}, \mathrm{b}$ ). These effects were significant even though time differences between the beginning and the end of the field study were only $38 \mathrm{~min}$ for sunrise and 19 min for sunset. Group size exerted an additional influence: the more birds per group the earlier the first song and the later the last one (Tables $2 a, b$ ).

Group size was also the main (and only significant) variable predicting the number of laugh songs at dusk. The more birds that congregated at the roost, the more often they sang (Table 2d). This is because normally each incoming Kookaburra elicits a new song. In two groups with five birds each, all group members roosted together on some nights, but split into two sub-groups of two and three birds on others. In the first case, they sang four to seven times, in the second case only one to two times. The number of songs at dawn depended on factors external to the group rather than internal ones like group size. The more neighbours surrounded a group the less the group members sang in the morning (Table $2 \mathrm{c}$ ). 
TABLE 2 Stepwise multiple regression analysis

\begin{tabular}{llllll}
\hline Variables & $\beta$ & $\alpha$ & $R^{2}$ & $\begin{array}{l}\text { d.f. } 1, \\
\text { d.f. } 2\end{array}$ & $P$
\end{tabular}

(a) FIRST SONG

$\begin{array}{lrrrrr}\text { group size } & -0.396 & 0.052 & 0.680 & 2,10 & 0.003 \\ \text { time of sunrise } & 0.695 & 0.003 & & & \end{array}$

$\begin{array}{llllll}\text { (b) LAST SONG } & & & & & \\ \text { group size } & 0.520 & 0.054 & 0.463 & 2,10 & 0.045 \\ \text { time of sunset } & 0.570 & 0.037 & & & \end{array}$

(c) \# DAWNSONGS

$\begin{array}{llllll}\text { \# of neighbours } & -0.747 & 0.013 & 0.559 & 1,8 & 0.013\end{array}$

(d) \# DUSKSONGS

group size $\quad 0.897<0.001 \quad 0.805 \quad 1,14<0.001$

Four song parameters (= dependent variables, in capital letters) were analysed in relation to demographic and ecological parameters ( $=$ independent variables). Standardised partial regression coefficients $(\beta)$ and their significance levels $(\alpha)$ are shown together with coefficients of determination $\left(R^{2}\right)$, degress of freedom ( $d f .1$, $d f .2$ ) and significance levels from the resulting regression model. $P$ differs from $\alpha$ only when more than one predictor variable is included in the model. Only independent variables with $\alpha<0.10$ were considered. For further explanations see text.

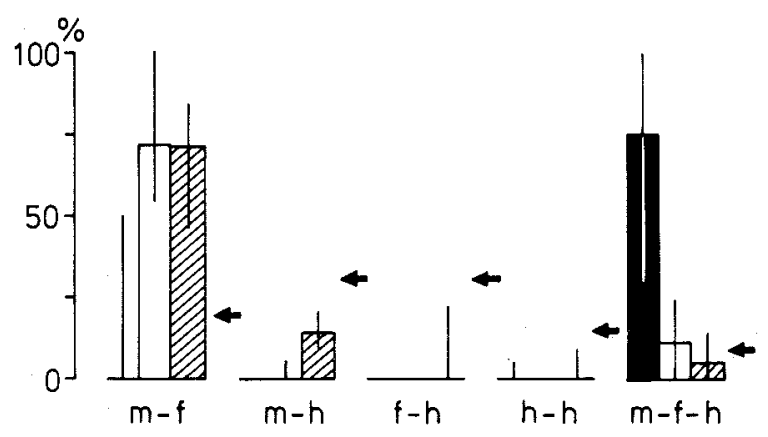

FIGURE 7 Percentage of songs at the roost (black bars) and during daytime (white bars) produced by five different combinations of birds. $m-f$ : breeding male and breeding female; $m$ $h$ : breeding male and 1-3 helpers; th: breeding female and - -3 helpers; h-h: $2-3$ helpers; m-f-h; breeding male, breeding female and $1-3$ helpers. Also shown are the percentages of the times the five combinations of birds were found perching together (hatched bars) and the expected frequencies of singing and perching together (arrows)

\section{Group composition during songs}

In pairs without helpers, singing can occur only between male and female, but in bigger groups five combinations are possible: $m-f, m-h, f-h, h-h, m-f-h$. These combinations only describe the types of birds involved, not their numbers. Therefore, depending on group size and situation, $\mathrm{f}-\mathrm{h}$ can stand for a mated female singing with one to three helpers and $h-h$ can denote singing between two to three helpers in groups with four or more birds, but cannot occur in groups of three.

It was noted, whenever possible, which birds in groups of three or more were involved in singing. The frequency in each of the five combinations was then expressed as the percentage of all songs in the respective group. Initially, these percent values were calculated separately for each of the following four catetories: (1) singing at dusk roost $(n$ $=8$ groups), (2) singing at dawn roost $(n=6)$, (3) unprovoked singing during daytime $(n=5)$ and (4) singing during daytime provoked by playback $(n=8)$. The relative frequencies of the five bird combinations differed neither between dusk and dawn, nor between unprovoked and provoked daytime songs (all $P \geq 0.206$; Mann-Whitney $U$ test). Therefore, categories (1) and (2) were merged into one data set for roost conditions and categories (3) and (4) into one for daytime conditions.

The laugh combination $m-f-h$, involving all types of group members, was the most frequent one at the roost where it occurred more often than during daytime (Fig. 7; $P=0.003$; Mann-Whitney U- test). The combination mf prevailed during daytime, when it was more frequent than at the roost $(P=0.001)$. The other three combinations ( $\mathrm{m}$ $\mathrm{h}, \mathrm{f}-\mathrm{h}, \mathrm{h}-\mathrm{h}$ ) were rare and their relative frequencies did not differ between roost and daytime conditions (all $P \geq$ 0.307 ).

Both the roost and the daytime distribution differed, however, from a random distribution based on the assumption that all group members are equally likely to laugh (Fig. 7; both $P=0.001$; Kolmogorov-Smirnov test, 2tailed). All possible combinations were considered when the expected distribution was calculated, i.e. $\mathrm{m}-\mathrm{hl}, \mathrm{m}-\mathrm{h} 2$, $\mathrm{m}-\mathrm{hl}-\mathrm{h} 2$, etc. Expectations were first calculated separately for groups of three, four and five birds. These individual expectations were then weighed by the proportion each group size was involved in singing to give one overall value for each of the five possible bird combinations shown in Figure 7 . At the roost, the m-f-h combination occurred more often than expected, during daytime this was true for the $\mathrm{m}-\mathrm{f}$ combination. All other associations fell either below expectation (m-h, $\mathrm{f}-\mathrm{h}, \mathrm{h}-\mathrm{h})$ or did not differ from it $(\mathrm{m}-\mathrm{f}-\mathrm{h})$.

\section{Spatial distribution when not singing}

During singing, Kookaburras usually sit in close proximity or at least are in visual contact with each other. However, perching together is not restricted to singing. It can also occur during resting, preening, foraging and other activi- 
ties. Whenever two or more non-singing birds were encountered on the same branch, it was noted who they were. It was also observed whether or not a bird followed when the other left the perch and flew to a different tree. These records were only taken during daytime and not at the roost, where poor light conditions usually prevented individual recognition.

The possible combinations of perching together are the same as for singing and their relative frequencies were calculated in the same way. The perching distribution differed significantly from a random distribution $(P=$ 0.030 ; Kolmogorov-Smirnov test, 2-tailed). Male and female were found together more often than expected; all other perching combinations either fell below the expected values $(m-h, f-h, h-h)$ or did not differ from them (m-f-h). This is the same as with singing and, in fact, there was no significant difference between the distributions of perching and daytime singing $(P=0.309)$.

The close association between male and female breeders, expressing itself in their frequent singing and perching together, is further supported by the proximity they keep during movements. When one mate changed trees the other usually followed immediately (median $94.5 \%$, interquartile range $=69.2-100 \%, n=6$ groups). This is significantly higher than in perching combinations involving helpers $(P=0.002$; Mann-Whitney U-test; data for $m-h, f-h, h-h$ and $m-f-h$ pooled). Here, movements of one bird - either mate or helper - normally elicited no following in the other (median $=0 \%$, interquartile range $=$ $0-28.6 \%, n=7$ groups). This lack of cohesion during movements is consistent with the lack of cohesion found in singing and perching.

\section{Laugh song and social interactions}

Although participation of helpers in singing is rare during daytime, it occurs occasionally and seems to be related to intra-group aggression. During our study, such aggressive behaviour expressed itself in chasing, pecking and sparring with clasped bills, usually accompanied by 'cackle' vocalisations (see Parry 1968, 1972 for detailed descriptions and illustrations). Upon hearing a helper sing, a mate, usually the male, would often fly straight towards him, push him from the branch or engage in a sparring match with him. Such attacks could also be provoked by playing a taperecorded song close to a silent helper.

The frequency of attacks per hour within groups was the only variable correlating positively with the proportion of daytime songs involving helpers $\left(r_{\mathrm{s}}=0.76, P<0.05, n=\right.$ 7 groups with $\geq 3$ birds; Spearman rank correlation, twotailed). Group size, number of neighbouring groups and total number of neighbouring birds did not correlate with helper laughing (all $r_{\mathrm{s}}<0.56, P>0.20$ ).
In the group with the highest proportion of helper laughing $(71 \%)$ and the highest attack rate $(0.88 / \mathrm{h})$, one helper, probably a male, was observed to regularly visit and inspect tree-holes with the female and also to (courtship?) feed her. In this group all attacks on the helper were carried out by the breeding male. In the only other group where 'nest-showing' and courtship feeding occurred regularly, they were restricted to the mated pair, which seems to be the normal pattern (Parry 1968). In this 'normal' group, helpers did not sing during daytime and the attack rate was only $0.09 / \mathrm{h}$. Moreover, in this latter group all five birds roosted together, whereas in the aggressive group one helper was expelled from the roost and spent the night separated from the others. These data and observations suggest that daytime singing by helpers may signal a challenge to a dominant mate's breeding status, leading to a destabilisation of the dominance hierarchy: In stable groups helpers apparently 'have little to laugh about'.

The close relationship between daytime laughing and aggression is also supported by observations on inter-group disputes, which take the form of chasing, 'trapeze flights' to the nest and 'circle flights' high above the territory (Parry 1968, 1972). This territorial advertisement could often be provoked by playbacks and was regularly accompanied by laugh songs. The laughing-aggression connection was also obvious in the group whose quarrels with neighbours led to deviations from the normal diurnal pattern of singing. The sequence of songs started after two neighbouring birds had trespassed on the territory and, after being chased by the owners, perched right at the boundary formed by a stream.

In one case, a Kookaburra repeatedly produced the actual laugh part of the song while attacking an Australian Raven Corvus coronoides. This again supports the aggressive nature of laughing, but in an interspecific context.

\section{Discussion}

According to Parry (1968, 1972) the Laughing Kookaburra's song serves four functions: (1) territorial defence and declaration; (2) localisation of group members for coordinating activities; (3) strengthening the social bond; and (4) relief of nervous tension. Parry's outline of point (4) is vague, but apparently she is talking about a motivational constraint rather then a real function. Therefore, this point will be not dealt with here.

Parry's evidence for the territorial function is threefold: (a) the song 'is most frequently given at or near the boundary together with defence display', (b) 'birds from neighbouring territories usually respond to it by laugh song' and (c) "its peak of intensity corresponds to the period of territorial adjustment' (Parry 1968, p. 34).

The data presented in our paper support (1), the territor- 
ial function idea and provide some additional evidence for it. However, we reject the proposed intra-group functions (2) and (3). Instead, we suggest that even in this context laughing has an aggressive meaning, which is related to mate-guarding and to establishing and maintaining a dominance hierarchy within the group. The hypothesis is based on the close association between laughing and aggression in all contexts, both between and within groups.

\section{Laughing and inter-group aggression}

The relationship between laughing and inter-group aggression became most obvious in the pair involved in a boundary dispute with trespassing neighbours. It is further supported by our finding that the number of dawn songs at the roost is not related to group size but to the number of neighbours (Table 2c). Perhaps surprisingly, song frequency decreased rather than increased with the number of neighbours. This may indicate that the laughing is mainly directed at vagrant birds that could be intruders. In a population with established territories, songs of all neighbours will add to the deterring effect. The more birds that are established the less each group has to contribute. This is similar to the frequently found decrease in individual vigilance as group size increases (e.g. Ward 1985). The deterring effect of songs probably does not only depend on the number of neighbours but also on the location of their territories, i.e. on the proportion of boundaries not 'buffered' by neighbours. Unfortunately, our small sample of plotted territories did not allow us to include this as an additional independent variable in the multiple regression model.

More support for the territorial function of laugh songs is their timing. Dawn songs constituted the highest proportion of all songs produced during a day (Fig. 6). This holds for many species of birds and is to be expected for the following reasons (Kacelnik \& Krebs 1983): (a) pressure on territory owners probably is highest in the early morning because intruders can expect some vacant territories due to overnight mortality; and (b) sound transmission is better in the early morning than later in the day. The importance of factor (b) can be assumed to increase with increasing distance between sender and receiver; i.e. transmission is probably more relevant for sounds directed to birds outside the territory than to members of the group.

The same argument holds for volume: signals to neighbours and vagrant birds should be louder than those to group members. As absolute volumes could not be measured and relative volumes will remain constant (Fig. 4) if all elements change by the same factor, we have no direct information on loudness of Kookaburra songs. There is, however, indirect information. Group songs not only lasted longer than pair songs, they also contained a higher proportion of the loudest element, the actual laugh part (Figs 2 and 4), while the proportions of the other elements either decreased or remained unchanged. Thus, group songs can be expected to reach farther for longer times. On the other hand, there was a positive correlation between group size and territory size as determined by the polygon method (Fig. 1). Although this method may not give the correct absolute territory sizes, especially when sample sizes are as small as in our study (MacDonald et al 1980), the error should not affect the correlation in a systematic way. Moreover, the correlation is consistent with that obtained by Parry (1973). She also showed that the number of group members determines territory size rather than vice versa (Table IV in Parry 1973).

These results suggest the following sequence of causal relationships: increasing group size increases song intensity which, in turn, aids in expanding the territory. Our present data, however, do not allow rigorous differentiation of this postulated cause-effect relationship from mere correlations between group size, song intensity and territory size.

\section{Laughing and intra-group aggression}

The relationship between laughing and intra-group aggression is mainly inferred from (1) the positive correlation between the frequency of intra-group attacks and the proportion of daytime songs involving helpers and (2) the fact that attacks on helpers could be provoked by song alone, be it from a real helper or played from a taperecorder.

In our study, all attacks were by the breeding pair on the helpers, supporting Parry's (1973) finding that the pair is dominant over all helpers. In some of our groups, the hierarchy appeared to be stable: attacks were rare, all birds roosted together and helpers did not participate in courtship feeding and nest inspection. In these groups helpers usually did not participate in daytime songs. In other groups, however, aggression was higher, groups split at the roost and helpers participated in feeding and nest visits. In these groups, helpers were also more frequently involved in daytime singing.

These results suggest that daytime singing by helpers may signal a challenge to the dominant mate's breeding status. Similar observations are available for a captive group of co-operatively breeding Striped Kingfishers $\mathrm{Hal}$ cyon chelicuti. A male young started copulating with his mother soon after he had started duetting with her, an activity that until then had been confined to his parents. This resulted in fights with his father and no further successful breeding in this previously very productive group (Reyer unpubl.). Thus, a clearcut dominance hierarchy seems to be essential for successful reproduction in co-operative breeders (see Reyer et al 1986 for further evidence and a more detailed discussion). In Laughing Kookaburras, the song may be a mild form of aggression 
that probably helps to establish and maintain that hierarchy.

This interpretation differs from Parry's view that intragroup laughing co-ordinates activities and strengthens the bond. The fact that laugh songs are a co-operative display by group members may have led her to describe their function in terms of the group's common interest - an unjustified conclusion often found in papers on duetting to which Parry refers (see Sonnenschein \& Reyer 1983 for a critical discussion). Common interest can be expected in some situations (e.g. in territorial defence) but not in all unless one assumes group selection, which is unlikely to occur either in Kookaburras or in most other species (Morton \& Parry 1974).

As each group member will tend to maximise its inclusive fitness, conflict between breeding male and breeding female and between parents and offspring is inevitable whenever their optimal strategies differ (Trivers 1972, 1974). In Kookaburras, signs of the parent-offspring conflict were found in aggressive interactions between breeders (= parents) and helpers (= offspring). These conflicts probably occur because helpers are barred from reproducing by the dominant breeders (Parry 1973).

Conflict between male and female breeders can be expected as a result of a male's tendency not only to guard his own female but also to attract other females, and of the female's opposing tendency to monopolise the parental investment of her mate (Trivers 1972; Yasukawa \& Searcy 1982). Sex-specificity in Kookaburra songs (Fig. 2) could enable males to acoustically court all females in the vicinity. In fact, it is primarily - although not exclusively - the male who initiates a song (Immelmann 1960; Parry 1968). Upon hearing his introductory calls, his own female will fly directly towards him, utter her own preliminaries (Immelmann 1960) and join the full laugh song after landing close to him. Combined with her sex-specific element, this could tell other females, 'Keep out, he is already paired!'. The same applies to songs started by the female (for whatever reason) and joined by the male. This interpretation, originally developed for duetting birds (Sonnenschein \& Reyer 1983), sees joint singing as acoustic mate-guarding, supplemented by physical guarding through close proximity of the singers. According to this hypothesis the frequent joint singing of Kookaburra male and female breeders does not reflect their common interest; rather, each answers the mate's song to prevent solosinging that could attract other birds as competitors. Although this has the effect of mates staying together, it is not what people usually mean when talking about 'strengthening the pair bond' as a function of a particular behaviour.

Our data, showing the close relationship between laughing and aggression, seem to support the above conflict hypotheses better than Parry's harmonious co-operation hypothesis.

\section{Overlap of different causes and functions}

The above differentiation between laughing in inter- and intra-group contexts may be an artificial one made by the human observer. For the bird all situations may be the same, namely aggressive interactions with the same causes and functions. A laughing helper, challenging the dominant breeder, may no longer be considered a group member but an intruding rival. Conversely, an intruding neighbour or vagrant bird may be considered a new group member that has to be incorporated into the dominance hierarchy.

This possible overlap between inter- and intra-group laughing should make us cautious about accepting some plausible explanations too readily. The fact that most songs occur during months when boundaries are adjusted (Parry 1968 ) is in itself no proof of territorial advertisement as the primary function. If mate attraction, pair formation and/or establishment of dominance hierarchies within groups coincide with territorial displays, then the same peak is to be expected even when song has no territorial function. Although quantitative data are lacking, there is some evidence for this: the rise in territorial defence, preceding the breeding season, seems to coincide with more vicious intra-group sparrings and dispersal of older helpers (Parry 1972, 1973). Consequently, potential sexual partners will be available. Any attempt by one mate to attract them should be met with mate-guarding by the other. Hence, and because of the changing hierarchies, song activity may increase.

A similar concurrence between inter- and intra-group contexts makes it difficult to interpret a second, smaller song peak when fledglings are present (Parry 1968, 1972 and Fig. 5 of this study). Parry suggests this localises dependent fledglings, which could be sitting anywhere in the territory. In our captive group, however, the young was always visible to the parents and laughing started only after they had stopped feeding him (Fig. 5). Also, the young start laughing only several weeks after becoming independent (Parry 1968), so the above explanation is unlikely. An alternative interpretation is that laughing after fledging is a means of integrating the new group members into the hierarchy. This is consistent with Parry's observation that parents and helpers frequently spar with fledglings. Another explanation is that the increase in song after breeding reflects a new breeding attempt. Signs of renewed breeding were observed in our captive group and second clutches do occasionally occur in the field (Parry 1973). For these new breeding attempts it could be beneficial to adjust territory boundaries to the new group size ( $c f$. Fig. 1) and/or attract other mates.

To distinguish between these and any other explanations 
would require a much longer and more detailed study than the one presented here. In our opinion, such a study should carry out investigations of the following:

(1) The motivations behind the visual and vocal behaviour patterns should be analysed. This may not only require methods used in classical motivation analyses (e.g. Toates 1986), but also endocrinological techniques such as radioimmunoassays and experimental application of hormones and hormone antagonists (e.g. Dittami \& Reyer 1984).

(2) The relationship between laughing and aggression should not be a mere correlation (as presented here) but a cause-effect connection. Analyses of behavioural transitions on a fine time scale would be one method to answer the question whether laughing is a cause and/or a consequence of aggressive behaviour.

(3) The effects and adaptive functions of laughing in inter- and intra-group social contexts should be studied. Here, multivariate approaches using variation in space and time within natural populations should be combined with studies on captive birds in which selected variables can be experimentally manipulated and controlled for.

\section{Acknowledgements}

The study was financed by the Max-Planck Institute, Seewiesen as part of a larger project on the behaviour and ecology of kingfishers. Additional funds for HUR's trip to Australia came from the DFG. We are grateful to W. Wickler for his interest in and support of the project and to the 'Vogelpark Walsrode' for making a pair of Kookaburras available to us. We would also like to thank Z. von Köckritz and L. Bals for their assistance in observing and caring, respectively, for the aviary birds. W. Arnold, D. Costa, J. Lamprecht, P. Ward, W. Wickler and two anonymous referees made helpful comments on an earlier version of the manuscript and L. Gardiner improved the English. Last, but not least, for their hospitality, HUR is grateful to all members of the Departments of Zoology and Ecosystem Management at the University of New England, Armidale, NSW, Australia, in particular to E. Wyndham who organised facilities for accommodation and transport.

\section{References}

Altmann, J. (1974). Observational study of behavior: sampling methods. Behaviour 49, 227-267.

Dittami, J.P. \& Reyer, H.-U. (1984). A factor analysis of seasonal, behavioral, hormonal and body weight changes in adult male barheaded geese, Anser indicus. Behaviour 90, 114124.

Immelmann, K. (1960). Behavioural observations on several species of Western Australian birds. Emu 60, 237-244.

Kacelnik, A. \& Krebs, J.R. (1983). The dawn chorus in the great tit (Parus major): proximate and ultimate causes. Behaviour 83, 287-309.

MacDonald, D.W., Ball, F.G. \& Hough, N.G. (1980). The evaluation of home range size and configuration using radio tracking data. In: A Handbook on Biotelemetry and Radio Tracking (eds C.J. Amlaner Jr. \& D.W. MacDonald) pp. 405-424. Pergamon Press, Oxford.

Morton, S.R. \& Parry, G.D. (1974). The auxiliary social system in Kookaburras: a reappraisal of its adaptive significance. Ети 74, 196-198.

Parry, V. (1968). Seasonality, territoriality and breeding biology of the Kookaburra, Dacelo gigas (Boddaert). M.Sc.thesis, Monash University, Clayton, Victoria, Australia.

Parry, V. (1972). Kookaburras. Taplinger, New York.

Parry, V. (1973). The auxiliary social system and its effect on territory and breeding in Kookaburras. Emu 73, 81-100.

Reyer, H.-U., Dittami, J.P. \& Hall, M.R. (1986). Avian helpers at the nest: are they psychologically castrated? Ethology 71, 216-228

Sachs, L. (1984). Angewandte Statistik, 6. Aufl. Springer-Verlag, Berlin.

Schmidl, D. (1985). Haltung und Zucht von Königsfischern (Alcedinidae). Gefiederte Welt 109, 118-121.

Sonnenschein, E. \& Heyer, H.-U. (1983). Mate-guarding and other functions of antiphonal duets in the slate-coloured boubou (Laniarius funebris). Z. Tierpsychol 63, 112-140.

Toates, F. (1986). Motivational Systems. Cambridge Univ. Press, Cambridge.

Trivers, R.L. (1972). Parental investment and sexual selection. In: Sexual Selection and the Descent of Man (ed. B. Campbell) pp. 136-179. Aldine, Chicago.

Trivers, R.L. (1974). Parent-offspring conflict. Am. Zool 14, 249 264.

Vehrencamp, S.L. (1983). A model for the evolution of despotic versus egalitarian societies. Anim. Behav. 31, 887-882.

Ward, P.I. (1885). Why birds in flocks do not coordinate their vigilance periods. J. Theor. Biol 114, 383-385.

Yasukawa, K. \& Searcy, W.A. (1982). Aggression in female redwinged blackbirds: a strategy to ensure male parental investment. Behav. Ecol. Sociobiol 11, 13-17. 